\title{
On restricted edge-connectivity of replacement product graphs*
}

\author{
HONG ZhenMu \\ School of Finance \\ Anhui University of Finance \& Economics \\ Bengbu, Anhui, 233030, China \\ Email: zmhong@mail.ustc.edu.cn \\ XU JunMing ${ }^{\dagger}$ \\ School of Mathematical Sciences \\ University of Science and Technology of China \\ Hefei, Anhui, 230026, China \\ Email: xujm@ustc.edu.cn
}

\begin{abstract}
This paper considers the edge-connectivity and the restricted edge-connectivity of replacement product graphs, gives some bounds on edge-connectivity and restricted edge-connectivity of replacement product graphs and determines the exact values for some special graphs. In particular, the authors further confirm that under certain conditions, the replacement product of two Cayley graphs is also a Cayley graph, and give a necessary and sufficient condition for such Cayley graphs to have maximum restricted edge-connectivity. Based on these results, the authors construct a Cayley graph with degree $d$ whose restricted edge-connectivity is equal to $d+s$ for given odd integer $d$ and integer $s$ with $d \geqslant 5$ and $1 \leqslant s \leqslant d-3$, which answers a problem proposed ten years ago.
\end{abstract}

Keywords: Graph theory, Connectivity, restricted edge-connectivity, replacement product, Cayley graph

AMS Subject Classification: $\quad 05 \mathrm{C} 40 \quad 68 \mathrm{M} 15 \quad 68 \mathrm{R} 10$

${ }^{*}$ This paper has been accepted for publication in SCIENCE CHINA Mathematics.

${ }^{\dagger}$ Corresponding author 


\section{Introduction}

Throughout this paper, we follow [35] for graph-theoretical terminology and notation not defined here. Specially, $G=(V, E)$ is a simple connected undirected graph, where $V=V(G)$ is the vertex-set of $G$ and $E=E(G)$ is the edge-set of $G ; d_{G}(x)$ is the degree of a vertex $x$ in $G$, the number of edges incident with $x$ in $G ; \delta(G)=\min \left\{d_{G}(x): x \in V(G)\right\}$ is the minimum degree of $G ; \xi(G)=\min \left\{d_{G}(x)+d_{G}(y)-2: x y \in E(G)\right\}$ is the minimum edge-degree of $G$.

The connectivity $\kappa(G)$ (resp. edge-connectivity $\lambda(G)$ ) of $G$ is defined as the minimum number of vertices (resp. edges) whose removal results in disconnected. The well-known Whitney inequality states that $\kappa(G) \leqslant \lambda(G) \leqslant \delta(G)$ for any graph $G$. In this paper, we are interested in the edge-connectivity $\lambda(G)$.

It is well known that when the underlying topology of an interconnection network is modeled by a connected graph $G=(V, E)$, where $V$ is the set of processors and $E$ is the set of communication links in the network, the edge-connectivity $\lambda(G)$ of $G$ is an important measurement for reliability and fault tolerance of the network since the larger $\lambda(G)$ is, the more reliable the network is. However, when computing $\lambda(G)$, one implicitly assumes that all edges incident with the same vertex may fail simultaneously. Consequently, this measurement is inaccurate for large-scale processing systems in which some subsets of system links can not fail at the same time in real applications.

To overcome the shortcomings of edge-connectivity, Esfahanian and Hakimi [5] proposed the concept of the restricted edge-connectivity $\lambda^{\prime}(G)$ of a graph $G$, which is the minimum number of edges whose removal results in disconnected and no isolated vertices, and gave the following result.

Theorem 1.1 (See [5]) $\quad \lambda(G) \leqslant \lambda^{\prime}(G) \leqslant \xi(G)$ for any graph $G$ of order $n(\geqslant 4)$ except for a star $K_{1, n-1}$.

A graph $G$ is vertex-transitive if for any two vertices $x$ and $y$ in $G$, there is a $\sigma \in$ Aut $(G)$ such that $y=\sigma(x)$, where $\operatorname{Aut}(G)$ is the automorphism group of $G$. Clearly, $\xi(G)=2 d-2$ for a vertex-transitive connected graph $G$ with degree $d$. Xu et al. obtained the following results.

Theorem 1.2 (See [37]) Let $G$ be a vertex-transitive connected graph with order $n(\geqslant$ 4) and degree $d(\geqslant 2)$. Then

(a) $\lambda^{\prime}(G)=\xi(G)=2 d-2$ if $n$ is odd or $G$ contains no triangles;

(b) there exists an integer $m(\geqslant 2)$ such that $d \leqslant \lambda^{\prime}(G)=\frac{n}{m} \leqslant 2 d-3$ otherwise.

Theorem 1.3 (See [18]) For any given integers $d$ and $s$ with $d \geqslant 3$ and $0 \leqslant s \leqslant d-3$, there is a connected vertex-transitive graph $G$ with degree $d$ and $\lambda^{\prime}(G)=d+s$ if and only if either $d$ is odd or $s$ is even.

In [18, for any odd integer $d(\geqslant 3)$ and any integer $s$ with $0 \leqslant s \leqslant d-3$, the authors construct a vertex-transitive graph $G$ with degree $d$ and $\lambda^{\prime}(G)=d+s=\frac{1}{2} n$. Note that the condition " $d \leqslant \lambda^{\prime}(G) \leqslant 2 d-3$ " implies $\lambda^{\prime}(G)=d$ if $d=3$. By Theorem 1.2, if a vertex-transitive graph $G$ is not $\lambda^{\prime}$-optimal, then $d \leqslant \lambda^{\prime}(G) \leqslant \frac{n}{2}$. Thus, a quite natural problem is proposed as follows (see Conjecture 1 in $\mathrm{Xu}[36]$ ). 
Problem 1.4 Given an odd integer $d(\geqslant 5)$ and any integer $s$ with $1 \leqslant s \leqslant d-3$, whether or not there is a vertex-transitive graph $G$ with order $n$ and degree $d$ such that $\lambda^{\prime}(G)=d+s<\frac{1}{2} n$.

In this paper, we answer this question confirmedly by constructing a Cayley graph, which is the replacement product of two Cayley graphs.

We will discuss the restricted edge-connectivity of a replacement product graph in this paper. The rest of this paper is organized as follows. In Section 2, we give some definitions with related results. In Section 3, we establish the bounds on the edge-connectivity for a replacement product graph and determine exact values under some special conditions. In Section 4, we give the lower and upper bounds on restricted edge-connectivity for replacement product graphs and determine exact values under some given conditions. In Section 5, we focus on Cayley graphs and further confirm that under certain conditions, the replacement product of two Cayley graphs is still a Cayley graph, and give a necessary and sufficient condition for such Cayley graphs to have maximum restricted edge-connectivity. Based on these results, we construct a Cayley graph to answer Problem 1.4 confirmedly. A conclusion is in Section 6.

\section{Preliminaries}

We first introduce the concept of the restricted edge-connectivity, proposed by Esfahanian and Hakimi [5], stated here slightly different from theirs.

Let $G$ be a non-trivial connected graph and $F \subset E(G)$. If $G-F$ is disconnected and contains no isolated vertices, then $S$ is called a restricted edge-cut of $G$. The restricted edge-connectivity of $G$, denoted by $\lambda^{\prime}(G)$, is defined as the minimum cardinality over all restricted edge-cuts of $G$. Esfahanian and Hakimi [5] proved $\lambda^{\prime}(G)$ is well-defined for any connected graph $G$ of order $n(\geqslant 4)$ except for a star $K_{1, n-1}$. A graph $G$ is $\lambda^{\prime}$-connected if $\lambda^{\prime}(G)$ exists, and a restricted edge-cut $F$ is a $\lambda^{\prime}$-cut if $|F|=\lambda^{\prime}(G)$. A $\lambda^{\prime}$-connected graph is $\lambda^{\prime}$-optimal if $\lambda^{\prime}(G)=\xi(G)$, and not $\lambda^{\prime}$-optimal otherwise. It is clear that if $G$ is a $\delta$-regular and $\lambda^{\prime}$-optimal graph of order $n$, then $\lambda(G)=\delta(G)=\delta$ and $n \geqslant 4$.

The restricted edge-connectivity provides a more accurate measure of fault-tolerance of networks than the edge-connectivity (see [4, 5]). Thus, determining the value of $\lambda^{\prime}$ for some special classes of graphs or characterizing $\lambda^{\prime}$-optimal graphs have received considerable attention in the literature (see, for instance, [5, 11, 12, 20, 23, 24, 31, 32, 33]).

Let $\Gamma$ be a finite group, and let $S$ be a subset of $\Gamma$ not containing the identity element of $\Gamma$. The Cayley graph $C_{\Gamma}(S)$ is the graph having vertex-set $\Gamma$ and edge-set $\left\{x y: x^{-1} y \in\right.$ $S, x, y \in \Gamma\}$.

Generally speaking, $C_{\Gamma}(S)$ is a digraph. The following result is well-known (see, for instance, $\mathrm{Xu}$ [34]).

Lemma 2.1 Cayley graphs are vertex-transitive and the Cartesian product of Cayley graphs is a Cayley graph.

If $S=S^{-1}$, then $C_{\Gamma}(S)$ is an undirected graph. We are interested in undirected graphs in this paper. 
We now introduce two classes of Cayley graphs, because of their excellent features, they are the most popular, versatile and efficient topological structures of interconnection networks (see, for instance, $\mathrm{Xu}[34]$ ).

Example 2.2 A circulant graph $G(n ; \pm S)$, where $S=\left\{s_{1}, s_{2}, \ldots, s_{k}\right\} \subseteq\{1,2, \ldots$, $\left.\left\lfloor\frac{1}{2} n\right\rfloor\right\}$ with $s_{1}<s_{2}<\ldots<s_{k}$ and $n \geqslant 3$, has vertex-set $V=\{0,1, \ldots, n-1\}$ and edge-set $E=\left\{i j:|j-i| \equiv s_{i}(\bmod n)\right.$ for some $\left.s_{i} \in S\right\}$.

Clearly, $G(n ; \pm 1)$ is a cycle $C_{n}$ and $G\left(n ; \pm\left\{1,2, \ldots,\left\lfloor\frac{1}{2} n\right\rfloor\right\}\right)$ is a complete graph $K_{n}$. The two graphs shown in Figure 1 are $G(8 ; \pm\{1,3\})$ and $G(8 ; \pm\{1,3,4\})$.

Note that the identity element of the $\operatorname{ring}$ group $\mathbb{Z}_{n}(n \geqslant 2)$ is just the zero element, and the inverse of any $i \in \mathbb{Z}_{n}$ is $n-i$. If let $S \subseteq\{1,2, \ldots, n-1\}$ and $S^{-1}=S$, then Cayley graph $C_{\mathbb{Z}_{n}}(S)$ is a circulant graph $G(n ; S)$ if $n \geqslant 3$, and $C_{\mathbb{Z}_{2}}(S)=K_{2}$. Thus, circulant graphs are vertex-transitive by Lemma 2.1 .

$\mathrm{Li}$ and $\mathrm{Li}$ [19] showed that $G(n ; \pm S)$ is $\lambda^{\prime}$-optimal and $\lambda^{\prime}(G(n ; \pm S))=4 k-2$ if $k \geqslant 2$ and $s_{k}<\frac{n}{2}$.
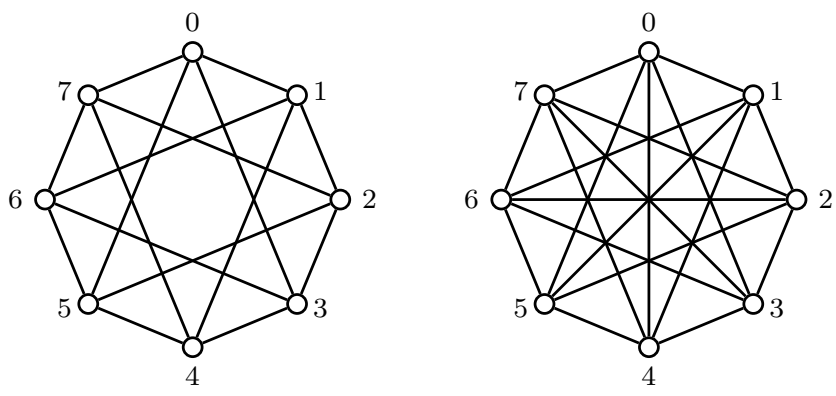

Figure 1: (a) $G(8 ; \pm\{1,3\})$; (b) $G(8 ; \pm\{1,3,4\})$

Example 2.3 The hypercube $Q_{n}$ has the vertex-set consisting of $2^{n}$ binary strings of length $n$, two vertices being linked by an edge if and only if they differ in exactly one position. Hypercubes $Q_{1}, Q_{2}, Q_{3}$ and $Q_{4}$ are shown in Figure 2.

It is easy to see that the hypercube $Q_{n}$ is Cartesian products $K_{2} \times K_{2} \times \cdots \times K_{2}$ of $n$ complete graph $K_{2}$. Let $\left(\mathbb{Z}_{2}\right)^{n}=\mathbb{Z}_{2} \times \mathbb{Z}_{2} \times \cdots \times \mathbb{Z}_{2}$ and

$$
e_{0}=\underbrace{0 \cdots 0}_{n} \text { and } e_{i}=\underbrace{0 \cdots 0}_{i-1} 1 \underbrace{0 \cdots 0}_{n-i} \text { for each } i=1,2, \ldots, n \text {. }
$$

Then $e_{0}$ is the identity element of $\left(\mathbb{Z}_{2}\right)^{n}$ and, by Lemma 2.1, $Q_{n}$ is a Cayley graph $C_{\left(\mathbb{Z}_{2}\right)^{n}}(S)$ and so is vertex-transitive, where $S=\left\{e_{1}, e_{2}, \cdots, e_{n}\right\}$, each of which is self-inverse and, hence, $S=S^{-1}$.

Esfahanian [4] showed that the hypercube $Q_{n}$ is $\lambda^{\prime}$-optimal, that is, $\lambda^{\prime}\left(Q_{n}\right)=2 n-2$ for $n \geqslant 2$.

Now, we introduce the replacement product. There are several equivalent definitions of the replacement product proposed by different authors (see [13, 28]). Here, we adopt the definition proposed by Hoory et al. [13]. Let $G_{1}$ be a $\delta_{1}$-regular graph on $n$ vertices and $G_{2}$ be a $\delta_{2}$-regular graph on $\delta_{1}$ vertices. For every vertex $x \in V\left(G_{1}\right)$, we label on all edges incident with $x$, say $e_{x}^{1}, e_{x}^{2}, \ldots, e_{x}^{\delta_{1}}$. 

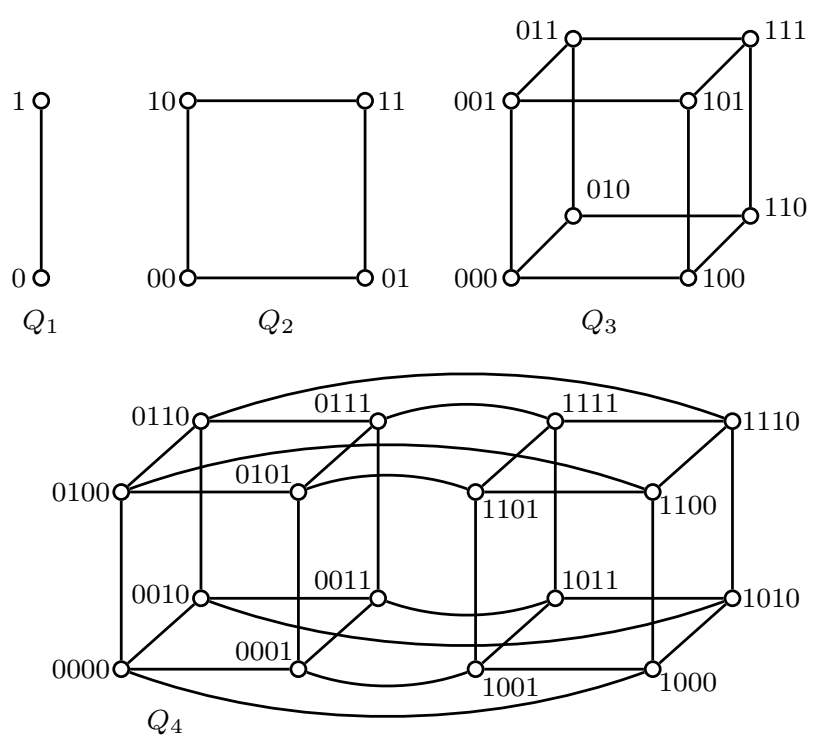

Figure 2: The $n$-cubes $Q_{1}, Q_{2}, Q_{3}$ and $Q_{4}$

Definition 2.4 Let $G_{1}$ be a $\delta_{1}$-regular graph on $n$ vertices and $G_{2}$ be a $\delta_{2}$-regular graph on $\delta_{1}$ vertices. The replacement product of $G_{1}$ and $G_{2}$ is a graph, denoted by $G_{1} \cap G_{2}$, where $V\left(G_{1} \AA G_{2}\right)=V\left(G_{1}\right) \times V\left(G_{2}\right)$, two distinct vertices $(x, i)$ and $(y, j)$, where $x, y \in$ $V\left(G_{1}\right)$ and $i, j \in V\left(G_{2}\right)$, are linked by an edge in $G_{1} \cap G_{2}$ if and only if either $x=y$ and $i j \in E\left(G_{2}\right)$, or $x y \in E\left(G_{1}\right)$ and $e_{x}^{i}=x y=e_{y}^{j}$.

Figure 3 shows the replacement product of $K_{4}$ and $C_{3}$ with given labelling of edges around vertices of $K_{4}$.

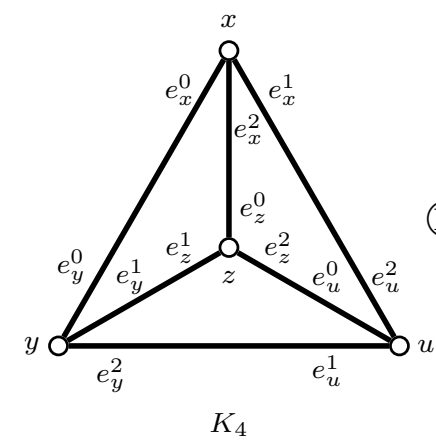

(B)
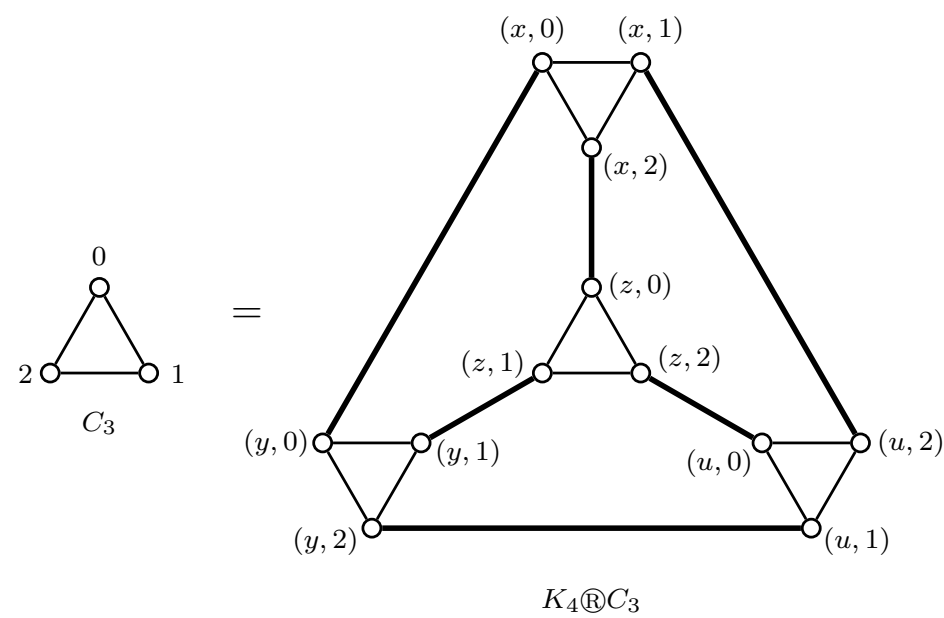

Figure 3: $K_{4}{ }^{\circledR} C_{3}$.

By Definition 2.4, we can obtain the following proposition.

Proposition 2.5 $\quad G_{1} \AA G_{2}$ is $\left(\delta_{2}+1\right)$-regular and has $n \delta_{1}$ vertices. Moreover, the vertex-set of $G_{1} \cap G_{2}$ can be partitioned into

$$
\left\{X_{1}, X_{2}, \ldots, X_{n}\right\} \text { such that } G\left[X_{i}\right] \cong G_{2} \text { for each } i \in I_{n} .
$$


The inflation or inflated graph of $G$ is a graph obtained from $G$ by replacing each vertex $x$ by a complete graph $K_{d_{G}(x)}$ and joining each edge to a different vertex of $K_{d_{G}(x)}$. Inflation graphs have been studied by several authors (for example, see [3, 6, 7, 14, 21, 27]). Clearly, if $G$ is $n$-regular then $G \AA K_{n}$ is the inflation graph of $G$. In special, Liu and Zhang [21] showed that $Q_{n} \circledast K_{n}$ is a Cayley graph.

The lexicographic product $G_{1}\left[G_{2}\right]$ of two graphs $G_{1}$ and $G_{2}$ is a graph with vertex-set $V\left(G_{1}\right) \times V\left(G_{2}\right)$, and in which two vertices $(x, i)$ and $(y, j)$ are adjacent if and only if either $x=y$ and $i j \in E\left(G_{2}\right)$ or $x y \in E\left(G_{1}\right)$, without the condition " $e_{x}^{i}=x y=e_{y}^{j}$ ". Thus, the replacement product graph $G_{1} 囚 G_{2}$ is a subgraph of the lexicographic product graph $G_{1}\left[G_{2}\right]$. In special, Li et al [17] showed that $G_{1}\left[G_{2}\right]$ is a Cayley graph if $G_{1}$ and $G_{2}$ are Cayley graphs.

The replacement product of two graphs is an important constructing method, which can obtain a larger graph from two smaller graphs, and so it has been widely used to address many fundamental problems in such areas as graph theory, combinatorics, probability, group theory, in the study of expander graphs and graph-based coding schemes [1, 2, 10, 13, 15, 16, 28]. The replacement product has been also used in the designing of an interconnection networks. For example, the well-known $n$-dimensional cube-connected cycle $C C C_{n}$ is a replacement product $Q_{n}{ }^{\circledR} C_{n}$, where $Q_{n}$ is a hypercube and $C_{n}$ is a cycle of length $n$ (see Preparata and Vuillemin [26]). The graph shown in Figure 4 is $Q_{3} 囚 C_{3}=C C C_{3}$. In addition, $n$-dimensional hierarchical hypercube is a replacement product $Q_{2^{n}} \cap Q_{n}$ (see Malluhi and Bayoumi [22]).
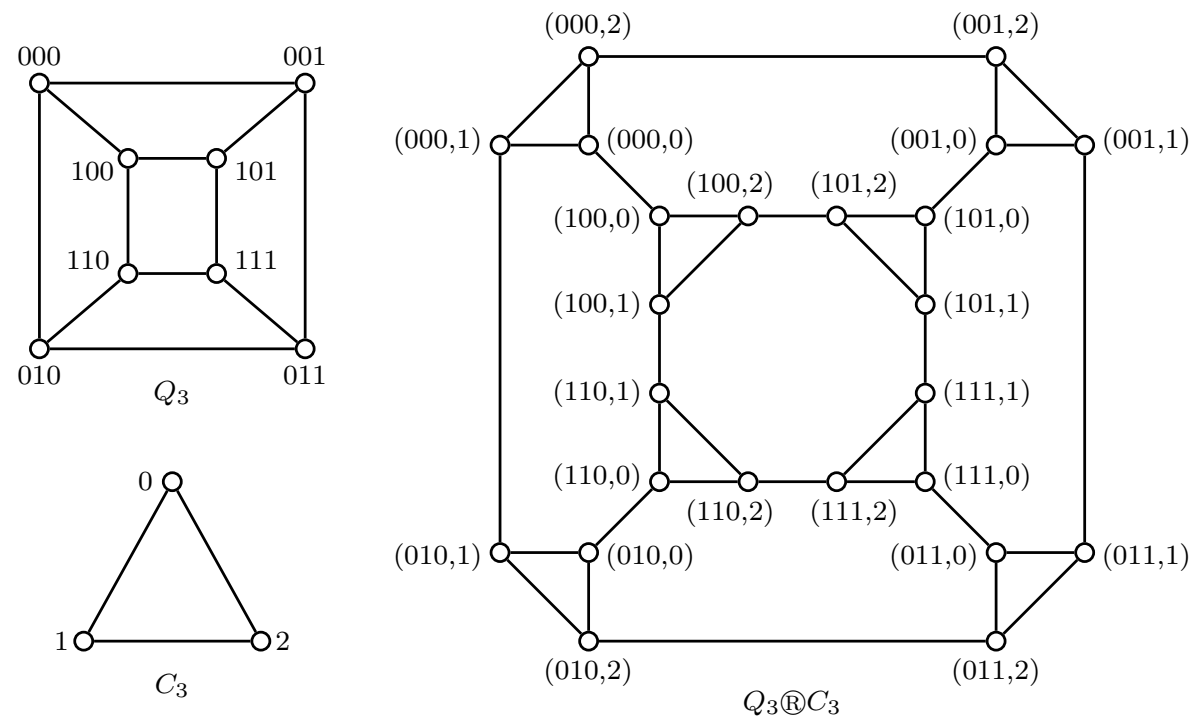

Figure 4: The cube-connected cycle $C C C(3)=Q_{3}{ }^{\circledR} C_{3}$.

For simplicity, when a replacement product graph $G_{1} \AA G_{2}$ is mentioned, if no otherwise specified, we always assume that $G_{1}$ is a $\delta_{1}$-regular graph with $n$ vertices and $G_{2}$ is a $\delta_{2}$ regular graph with $\delta_{1}$ vertices. Moreover, we simply write $\kappa_{i}=\kappa\left(G_{i}\right), \lambda_{i}=\lambda\left(G_{i}\right)$ and $\delta_{i}=\delta\left(G_{i}\right)$ for each $i=1,2$, and write $x G_{2}$ for $\{x\} \times G_{2}$ for any $x \in V\left(G_{1}\right)$, and let $I_{n}=\{1,2, \ldots, n\}$. 
In this paper, we also need some notations. For a subset $X \subset V(G)$, use $G[X]$ to denote the subgraph of $G$ induced by $X$. For two disjoint subsets $X$ and $Y$ in $V(G)$, use $[X, Y]$ to denote the set of edges between $X$ and $Y$ in $G$. In particular, $E_{G}(X)=[X, \bar{X}]$ and let $d_{G}(X)=\left|E_{G}(X)\right|$, where $\bar{X}=V(G) \backslash X$.

For a $\lambda^{\prime}$-connected graph $G$, there is certainly a subset $X \subset V(G)$ with $|X| \geqslant 2$ such that $E_{G}(X)$ is a $\lambda^{\prime}$-cut and, both $G[X]$ and $G[\bar{X}]$ are connected. Such an $X$ is called a $\lambda^{\prime}$-fragment of $G$. A $\lambda^{\prime}$-fragment $X$ of $G$ with minimum cardinality is called a $\lambda^{\prime}$-atom of $G$. The $\lambda^{\prime}$-atom has been successfully used in the study of restricted edge-connectivity of graphs (see, for instance, [23, 25, 30, 37]).

\section{Edge-connectivity of $G_{1} \circledast G_{2}$}

In this section, we investigate the edge-connectivity of replacement product graph $G_{1} 囚 G_{2}$. By Definition 2.4, it is easy to see that if $G_{1}$ and $G_{2}$ are connected, then $G_{1} \AA G_{2}$ is also connected. We now establish the upper and lower bounds on the edge-connectivity for replacement product graphs.

Theorem 3.1 If both $G_{1}$ and $G_{2}$ are connected, then

$$
\min \left\{\lambda_{1}, \lambda_{2}\right\} \leqslant \lambda\left(G_{1} 囚 G_{2}\right) \leqslant \min \left\{\lambda_{1}, \delta_{2}+1\right\} .
$$

Furthermore,

$$
\min \left\{\lambda_{1}, \lambda_{2}+1\right\} \leqslant \lambda\left(G_{1} \cap G_{2}\right) \text { if } \kappa_{1} \geqslant 2 .
$$

Proof. Let $G=G_{1} \AA G_{2}$. Clearly,

$$
\lambda(G) \leqslant \delta(G)=\delta_{2}+1
$$

Let $S \subset V\left(G_{1}\right)$ and $E_{G_{1}}(S)$ be a $\lambda_{1}$-cut of $G_{1}$, and $T=\left\{(x, i): x \in S, i \in V\left(G_{2}\right)\right\}$. Then $E_{G}(T)$ is an edge-cut of $G$. Since there is an edge $x y$ in $G_{1}$ if and only if there is exactly one edge between $V\left(x G_{2}\right)$ and $V\left(y G_{2}\right)$ in $G, x y \in E_{G_{1}}(S)$ if and only if there are two vertices $i$ and $j$ of $G_{2}$ such that $((x, i),(y, j)) \in E_{G}(T)$. Therefore, $\left|E_{G}(T)\right|=$ $\left|E_{G_{1}}(S)\right|=\lambda_{1}$ and

$$
\lambda(G) \leqslant\left|E_{G}(T)\right|=\lambda_{1} .
$$

Combining (3.3) with (3.4), we establish the upper bound on $\lambda\left(G_{1} \cap G_{2}\right)$ in (3.1). We now show the lower bound in (3.1).

Let $F$ be a $\lambda$-cut in $G$. Then there are two $\lambda$-fragments associated with $F$ in $G$, say, $X$ and $\bar{X}$. Let $\left\{V_{1}, V_{2}, \ldots, V_{n}\right\}$ be a partition of $V(G)$ satisfied property in Proposition 2.5.

Assume for each $i \in I_{n}$, either $V_{i} \subset X$ or $V_{i} \subset \bar{X}$. Let $Y=\left\{i: V_{i} \subset X, i \in I_{n}\right\}$. Then $Y \subset V\left(G_{1}\right), E_{G_{1}}(Y)$ is an edge-cut of $G_{1}$ and $\left|E_{G_{1}}(Y)\right|=|F|$, and so

$$
\lambda(G)=|F|=\left|E_{G_{1}}(Y)\right| \geqslant \lambda_{1} .
$$

Assume now that there exists some $i \in I_{n}$ such that $V_{i} \cap X \neq \emptyset$ and $V_{i} \cap \bar{X} \neq \emptyset$. Then

$$
\lambda(G)=|F| \geqslant\left|\left[V_{i} \cap X, V_{i} \cap \bar{X}\right]\right| \geqslant \lambda\left(G\left[V_{i}\right]\right)=\lambda\left(G_{2}\right)=\lambda_{2} .
$$


Combining (3.5) with (3.6) , we establish the lower bound on $\lambda\left(G_{1} \AA G_{2}\right)$ in (3.1).

To prove (3.2), let $(x, i)$ be any vertex of $V_{x} \cap X$ and $(x, j)$ be any vertex of $V_{x} \cap \bar{X}$. Since $G\left[V_{x}\right] \cong G_{2}$ and $G_{2}$ is $\lambda_{2}$-connected, there exist $\lambda_{2}$ edge-disjoint paths $P_{1}, P_{2}, \ldots, P_{\lambda_{2}}$ between $(x, i)$ and $(x, j)$ in $G\left[V_{x}\right] \subset G$. Let $(y, k) \in N_{G}((x, i))$ and $(z, \ell) \in N_{G}((x, j))$, where $\{y, z\} \subseteq N_{G_{1}}(x)$. Since $\kappa_{1} \geqslant 2$, there exist at least two internally vertex-disjoint paths between $y$ and $z$ in $G_{1}$, one of them avoids $x$. By the connectedness of $G_{2}$, there exists a path $Q$ between $(y, k)$ and $(z, \ell)$ in $G$ that avoids the vertices of $V_{x}$. Let $P_{0}=$ $\langle(x, i), Q,(x, j)\rangle$. Thus, $P_{0}, P_{1}, P_{2}, \ldots, P_{\lambda_{2}}$ are $\lambda_{2}+1$ edge-disjoint paths between $(x, i)$ and $(x, j)$. Since $(x, i) \in V_{x} \cap X$ and $(x, j) \in V_{x} \cap \bar{X}$, it is easy to find $\left|E\left(P_{i}\right) \cap F\right| \geqslant 1$ for each $i \in\left\{0,1,2, \ldots, \lambda_{2}\right\}$ and so

$$
\lambda(G)=|F| \geqslant \lambda_{2}+1
$$

as required.

Combining the Whitney's inequality $\kappa(G) \leqslant \lambda(G) \leqslant \delta(G)$ with Theorem [3.1, we obtain the following results immediately.

Corollary 3.2 Suppose that both $G_{1}$ and $G_{2}$ are connected. Then

(a) $\lambda\left(G_{1} 囚 G_{2}\right)=1$ if $\lambda_{1}=1$;

(b) $\lambda\left(G_{1} 囚 G_{2}\right)=\lambda_{1}$ if $\lambda_{2} \geqslant \lambda_{1}$;

(c) $\lambda\left(G_{1} \AA G_{2}\right)=\lambda_{1}$ if $\kappa_{1} \geqslant 2$ and $\lambda_{2} \geqslant \lambda_{1}-1$;

(d) $\lambda\left(G_{1} 囚 G_{2}\right)=\min \left\{\lambda_{1}, \delta_{2}+1\right\}$ if $\kappa_{1} \geqslant 2$ and $\lambda_{2}=\delta_{2}$.

Lemma 3.3 $\lambda(G) \leqslant \frac{1}{2} \Delta(G)$ for any connected graph that contains cut-vertices.

Proof. Suppose that $x$ is a cut-vertex of $G$ and $G-x$ has $k$ components, where $k \geqslant 2$. Then $\lambda(G) \leqslant \frac{1}{k}|N(x)| \leqslant \frac{1}{2} \Delta(G)$.

Corollary 3.4 $\lambda\left(G \AA K_{n}\right)=\lambda(G)$ for any $n$-regular connected graph $G$.

Proof. Clearly, $\lambda(G) \leqslant \delta(G)=n$ and $\lambda\left(K_{n}\right)=\delta\left(K_{n}\right)=n-1$. If $\kappa(G) \geqslant 2$, then $\lambda\left(G \AA K_{n}\right)=\lambda(G)$ by Theorem 3.1. If $\kappa(G)=1$, by Lemma 3.3, then $\lambda(G) \leqslant \frac{n}{2}<n$ and so $\lambda\left(K_{n}\right) \geqslant \lambda(G)$. By Corollary 3.2 (b), the result follows.

Corollary 3.5 $\lambda\left(G \AA C_{n}\right)=\min \{\lambda(G), 3\}$ for any 2-connected $n$-regular graph $G$.

Example 3.6 $\lambda\left(K_{4} 囚 C_{3}\right)=\lambda\left(K_{4}\right)=3$, and

$$
\lambda\left(C C C_{n}\right)=\lambda\left(Q_{n} 囚 C_{n}\right)=\min \left\{\lambda\left(Q_{n}\right), 3\right\}=\min \{n, 3\}=3 \text { if } n \geqslant 3 .
$$

Remark 3.7 We conclude this section with a remark on Theorem 3.1. The condition " $\kappa_{1} \geqslant 2$ " in (3.2) is necessary. For example, two graphs $G_{1}$ and $G_{2}$ are shown in Figure 5 . It is easy to see that $\kappa_{1}=1, \lambda_{1}=4$, and $\lambda_{2}=\delta_{2}=2, G_{1} \AA G_{2}$ is 3-regular, and

$$
\lambda\left(G_{1} 囚 G_{2}\right)=2<\min \{4,3\}=\min \left\{\lambda_{1}, \lambda_{2}+1\right\},
$$

which contradicts to the lower bound on $\lambda\left(G_{1} 囚 G_{2}\right)$ given in (3.2). 

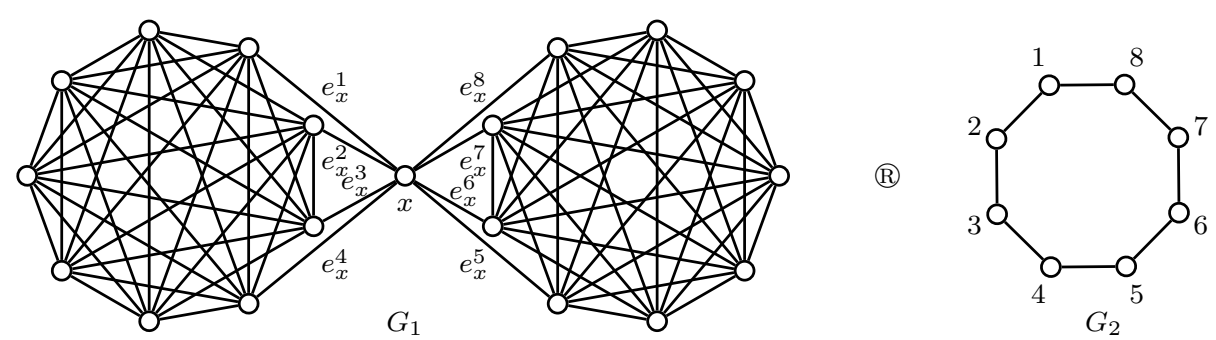

Figure 5: Two graphs $G_{1}$ and $G_{2}$ in Remark 3.7.

\section{Restricted edge-connectivity of $G_{1} \circledast G_{2}$}

In this section, we investigate the restricted edge-connectivity of the replacement product of two regular graphs.

Theorem 4.1 If both $G_{1}$ and $G_{2}$ are connected, then

$$
\lambda^{\prime}\left(G_{1} 囚 G_{2}\right) \leqslant \min \left\{\lambda_{1}, 2 \delta_{2}\right\} .
$$

Proof. Let $G=G_{1} \AA G_{2}$. Since $G$ is $\left(\delta_{2}+1\right)$-regular and $\delta_{2}+1 \geqslant 2$, it is easy to see that $G$ is $\lambda^{\prime}$-connected. By Theorem 1.1.

$$
\lambda^{\prime}(G) \leqslant \xi(G)=2 \delta_{2} .
$$

Let $X \subset V\left(G_{1}\right)$ such that $[X, \bar{X}]_{G_{1}}$ be a $\lambda_{1}$-cut of $G_{1}$. Then $G_{1}[X]$ and $G_{1}[\bar{X}]$ are both connected. Let $Y=\left\{(x, i): x \in X, i \in V\left(G_{2}\right)\right\}$. Then $G[Y]$ and $G[\bar{Y}]$ are connected, and $|Y| \geqslant\left|V\left(G_{2}\right)\right|=\delta_{1} \geqslant 2,|\bar{Y}| \geqslant\left|V\left(G_{2}\right)\right|=\delta_{1} \geqslant 2$. Hence, $[Y, \bar{Y}]_{G}$ is a restricted edge-cut of $G$. There is an edge $x y$ in $G_{1}$ if and only if there is exactly one edge between $V\left(x G_{2}\right)$ and $V\left(y G_{2}\right)$ in $G$, so $x y \in[X, \bar{X}]_{G_{1}}$ if and only if there are two vertices $i$ and $j$ of $G_{2}$ such that $((x, i),(y, j)) \in[Y, \bar{Y}]_{G}$. Therefore, $\left|[Y, \bar{Y}]_{G}\right|=\left|[X, \bar{X}]_{G_{1}}\right|=\lambda_{1}$ and

$$
\lambda^{\prime}(G) \leqslant\left|[Y, \bar{Y}]_{G}\right|=\lambda_{1} \text {. }
$$

Combining (4.1) with (4.2), the result follows.

Theorem 4.2 $\quad \lambda^{\prime}\left(G 囚 K_{n}\right)=\lambda(G)$ for any $n$-regular connected graph $G$.

Proof. By Corollary [3.4, $\lambda\left(G \AA K_{n}\right)=\lambda(G)$. By Theorem 4.1, $\lambda^{\prime}\left(G \AA K_{n}\right) \leqslant \lambda(G)$, and so

$$
\lambda(G)=\lambda\left(G \AA K_{n}\right) \leqslant \lambda^{\prime}\left(G \AA K_{n}\right) \leqslant \lambda(G) .
$$

The result follows.

For $\delta_{1} \leqslant 3$, Theorem 4.2 shows that $\lambda^{\prime}\left(G_{1} \cap G_{2}\right)=\lambda\left(G_{1}\right)$. In the following discussion, we always assume $\delta_{1} \geqslant 4$.

Lemma 4.3 Suppose that both $G_{1}$ and $G_{2}$ are connected and $\delta_{1} \geqslant 4, F$ be a $\lambda^{\prime}$-cut of $G_{1} \AA G_{2}$ and $\left\{X_{1}, X_{2}, \ldots, X_{n}\right\}$ be a partition of $V\left(G_{1} \circledast G_{2}\right)$ satisfied property in Proposition [2.5. If there is some $i \in I_{n}$ such that $G\left[X_{i}\right]$ is disconnected in $G-F$, then

$$
\lambda^{\prime}\left(G_{1} 囚 G_{2}\right) \geqslant \min \left\{\kappa_{1}+\lambda_{2}-1,2 \lambda_{2}, \lambda_{2}^{\prime}+2\right\} .
$$


Proof. Let $G=G_{1} \AA G_{2}$. Since $F$ is a $\lambda^{\prime}$-cut of $G$, there is some $X \subset V(G)$ with $|X| \geqslant 2$ such that $F=E_{G}(X)$. Without loss of generality assume $|\bar{X}| \geqslant|X|$.

If there exist two distinct $j, k \in I_{n}$ such that $G\left[X_{j}\right]$ and $G\left[X_{k}\right]$ are disconnected in $G-F$, then

$$
|F| \geqslant \lambda\left(G\left[X_{j}\right]\right)+\lambda\left(G\left[X_{k}\right]\right)=2 \lambda_{2} .
$$

Now assume that there exists exactly one integer, say $j \in I_{n}$, such that $G\left[X_{j}\right]$ is disconnected in $G-F$. Then $X_{j} \cap X \neq \emptyset$ and $X_{j} \cap \bar{X} \neq \emptyset$. Consider the following two cases.

Case 1. $X \subset X_{j}$.

In this case, $\bar{X}=\left(V(G) \backslash X_{j}\right) \cup\left(X_{j} \backslash X\right)$. Thus

$$
\begin{aligned}
|F| & =|[X, \bar{X}]| \\
& =\left|\left[X,\left(V(G) \backslash X_{j}\right)\right]\right|+\left|\left[X, X_{j} \backslash X\right]\right| \\
& =|X|+\left|\left[X, X_{j} \backslash X\right]\right|
\end{aligned}
$$

If $|X|=\delta_{1}-1$, then $\left|\left[X, X_{j} \backslash X\right]\right|=\delta_{2}$, and so

$$
|F|=\delta_{1}-1+\delta_{2} \geqslant 2 \delta_{2} \geqslant 2 \lambda_{2} .
$$

If $2 \leqslant|X| \leqslant \delta_{1}-2$, then $\left[X, X_{j} \backslash X\right]$ is a restricted edge-cut of $G\left[X_{j}\right]$, and so

$$
|F| \geqslant|X|+\lambda^{\prime}\left(G\left[X_{j}\right]\right) \geqslant \lambda_{2}^{\prime}+2 \text {. }
$$

Hence, in this case,

$$
|F| \geqslant \min \left\{2 \lambda_{2}, \lambda_{2}^{\prime}+2\right\}
$$

Case 2. $X \nsubseteq X_{j}$.

Since $|\bar{X}| \geqslant|X|, \bar{X} \nsubseteq X_{j}$. Equivalently, there exist at least two sets $X_{k}$ and $X_{\ell}$ other than $X_{j}$ such that $X_{k} \subset X$ and $X_{\ell} \subset \bar{X}$. Since $\kappa\left(G_{1}-u\right) \geqslant \kappa_{1}-1 \geqslant 0$ for any vertex $u \in V\left(G_{1}\right)$, there are at least $\kappa_{1}-1$ internally vertex-disjoint paths between any two distinct vertices $x$ and $y$ in $G_{1}-u$. By the definition of $G$, it is easy to see that there are at least $\kappa_{1}-1$ internally vertex-disjoint paths $P_{1}, P_{2}, \ldots, P_{\kappa_{1}-1}$ between $X_{k}$ and $X_{\ell}$ in $G-X_{j}$. Let $F^{\prime}=F \backslash\left[X_{j} \cap X, X_{j} \cap \bar{X}\right]$. Since $X_{k} \subset X$ and $X_{\ell} \subset \bar{X},\left|E\left(P_{i}\right) \cap F^{\prime}\right| \geqslant 1$ for each $i \in\left\{1,2, \ldots, \kappa_{1}-1\right\}$ and $\left|F^{\prime}\right| \geqslant \kappa_{1}-1$. Thus, we have

$$
\begin{aligned}
|F|=|[X, \bar{X}]| & =\left|F^{\prime}\right|+\left|\left[X_{j} \cap X, X_{j} \cap \bar{X}\right]\right| \\
& \geqslant \kappa_{1}-1+\lambda\left(G\left[X_{j}\right]\right) \\
& =\kappa_{1}+\lambda_{2}-1
\end{aligned}
$$

that is,

$$
|F| \geqslant \kappa_{1}+\lambda_{2}-1
$$

Note that if $\kappa_{1}=1$, then $|F| \geqslant\left|\left[X_{j} \cap X, X_{j} \cap \bar{X}\right]\right| \geqslant \lambda_{2}$ and so (4.6) also holds.

By (4.4), (4.5) and (4.6), the inequality (4.3) is established. 
Theorem 4.4 Suppose that both $G_{1}$ and $G_{2}$ are connected and $\delta_{1} \geqslant 4$. Then

$$
\min \left\{\lambda_{1}, \kappa_{1}+\lambda_{2}-1,2 \lambda_{2}, \lambda_{2}^{\prime}+2\right\} \leqslant \lambda^{\prime}\left(G_{1} \mathbb{R} G_{2}\right) \leqslant \min \left\{\lambda_{1}, 2 \delta_{2}\right\} .
$$

Furthermore, if $\kappa_{1} \geqslant \lambda_{1}-\lambda_{2}+1$ (or $\kappa_{1} \geqslant \lambda_{2}+1$ ) and $G_{2}$ is $\lambda^{\prime}$-optimal, then

$$
\lambda^{\prime}\left(G_{1} 囚 G_{2}\right)=\min \left\{\lambda_{1}, 2 \delta_{2}\right\} .
$$

Proof. Let $G=G_{1} 囚 G_{2}$. By Theorem 4.1, we only need to show the lower bound on $\lambda^{\prime}\left(G_{1} \AA G_{2}\right)$ in (4.7). To the end, let $\left\{X_{1}, X_{2}, \ldots, X_{n}\right\}$ be a partition of $V(G)$ satisfied property in Proposition 2.5 and $F$ be a $\lambda^{\prime}$-cut of $G$. There is some $X \subset V(G)$ with $|X| \geqslant 2$ such that $F=E_{G}(X)$. Without loss of generality assume $|\bar{X}| \geqslant|X|$.

By Lemma 4.3, we only need to show that $\lambda^{\prime}(G) \geqslant \lambda_{1}$ if $G\left[X_{i}\right]$ is connected in $G-F$ for each $i \in I_{n}$.

In this case, either $X_{i} \subset X$ or $X_{i} \subset \bar{X}$ for each $i \in I_{n}$. Thus, we can assume $F=E_{G}\left(Y \times V\left(G_{2}\right)\right)$, where $Y \subset V\left(G_{1}\right)$. By the definition of $G,|F|=\left|E_{G}\left(Y \times V\left(G_{2}\right)\right)\right|=$ $\left|E_{G_{1}}(Y)\right|$. Since $\left|E_{G_{1}}(Y)\right| \geqslant \lambda_{1}$, we have $|F| \geqslant \lambda_{1}$, and so the lower bound on $\lambda^{\prime}\left(G_{1} 囚 G_{2}\right)$ in (4.7) is established.

We now show the equality (4.8). If $\kappa_{1} \geqslant \lambda_{1}-\lambda_{2}+1$ (or $\kappa_{1} \geqslant \lambda_{2}+1$ ) and $G_{2}$ is $\lambda^{\prime}$-optimal, then $\lambda_{2}^{\prime}=\xi\left(G_{2}\right)=2 \delta_{2}-2$, and so $\lambda_{2}=\delta_{2}$. Thus, we have

$$
\kappa_{1}+\lambda_{2}-1 \geqslant \lambda_{1}\left(\text { or } \kappa_{1}+\lambda_{2}-1 \geqslant 2 \lambda_{2}\right),
$$

and so

$$
\min \left\{\lambda_{1}, \kappa_{1}+\lambda_{2}-1,2 \lambda_{2}, \lambda_{2}^{\prime}+2\right\}=\min \left\{\lambda_{1}, 2 \delta_{2}\right\} .
$$

Comparing (4.7) with (4.9), the equality (4.8) is established.

Note that if $G_{2}$ is $\lambda^{\prime}$-optimal then $\delta_{1}=\left|V\left(G_{2}\right)\right| \geqslant 4$, and so $\lambda^{\prime}\left(G_{1} \AA G_{2}\right)$ is well-defined. By Theorem 4.4, we obtain the following corollary immediately.

Corollary 4.5 Assume $G_{1}$ and $G_{2}$ are connected. If $\kappa_{1}=\lambda_{1}$ and $G_{2}$ is $\lambda^{\prime}$-optimal, then

$$
\lambda^{\prime}\left(G_{1} 囚 G_{2}\right)=\min \left\{\lambda_{1}, 2 \delta_{2}\right\} .
$$

A connected graph $G$ is super- $\lambda$ if every $\lambda$-cut isolates a vertex in $G$. It is clear that $G$ is super- $\lambda$ if and only if $\lambda^{\prime}(G)>\lambda(G)$. By Theorem 4.4, we obtain the following results immediately.

Theorem 4.6 Suppose that $G_{1}$ and $G_{2}$ are two connected graphs. If $\kappa_{1} \geqslant \lambda_{1}-\lambda_{2}+1 \geqslant 2$ (or $\kappa_{1} \geqslant \lambda_{2}+1$ ) and $G_{2}$ is $\lambda^{\prime}$-optimal, then

(a) $G_{1} \AA G_{2}$ is $\lambda^{\prime}$-optimal if and only if $\lambda_{1} \geqslant 2 \delta_{2}$;

(b) $G_{1} \cap G_{2}$ is super- $\lambda$ if and only if $\lambda_{1}>\delta_{2}+1$.

Proof. Let $G=G_{1} 囚 G_{2}$. Clearly, $\xi(G)=2 \delta_{2}$, and $\lambda_{2}=\delta_{2} \geqslant 2$ since $G_{2}$ is $\lambda^{\prime}$-optimal.

Since $\kappa_{1} \geqslant \lambda_{1}-\lambda_{2}+1$ (or $\kappa_{1} \geqslant \lambda_{2}+1$ ) and $G_{2}$ is $\lambda^{\prime}$-optimal, by Theorem 4.4 we have that

$$
\lambda^{\prime}(G)=\min \left\{\lambda_{1}, 2 \delta_{2}\right\} .
$$


Thus, $G$ is $\lambda^{\prime}$-optimal if and only if $\lambda^{\prime}(G)=\xi(G)=2 \delta_{2}$, that is $\lambda_{1} \geqslant 2 \delta_{2}$ from (4.10).

Since $\kappa_{1} \geqslant 2$ and $\lambda_{2}=\delta_{2}$, by Corollary 3.2 (d) we have that

$$
\lambda(G)=\min \left\{\lambda_{1}, \delta_{2}+1\right\} .
$$

Note $2 \delta_{2}>\delta_{2}+1$ for $\delta_{2} \geqslant 2$. It follows that $G$ is super- $\lambda$ if and only if $\lambda^{\prime}(G)>\lambda(G)$, that is $\lambda_{1}>\delta_{2}+1$ from (4.11).

Corollary 4.7 Assume $G_{1}$ and $G_{2}$ are two connected graphs with $\delta_{1} \geqslant 4$. If $\kappa_{1}=\lambda_{1} \geqslant$ 2 and $G_{2}$ is $\lambda^{\prime}$-optimal, then

(a) $G_{1} 囚 G_{2}$ is $\lambda^{\prime}$-optimal if and only if $\lambda_{1} \geqslant 2 \delta_{2}$;

(b) $G_{1} \cap G_{2}$ is super- $\lambda$ if and only if $\lambda_{1}>\delta_{2}+1$.

Corollary 4.8 $\quad \lambda^{\prime}\left(G \AA C_{n}\right)=\min \{\lambda(G), 4\}$ if $G$ is an $n$-regular graph and $\kappa(G) \geqslant 3$.

Example 4.9 By Corollary 4.8, it is easy to see that $\lambda^{\prime}\left(K_{4} 囚 C_{3}\right)=\min \left\{\lambda\left(K_{4}\right), 4\right\}=\min \{3,4\}=3$, and $\lambda^{\prime}\left(C C C_{n}\right)=\lambda^{\prime}\left(Q_{n} 囚 C_{n}\right)=\min \left\{\lambda\left(Q_{n}\right), 4\right\}=\min \{n, 4\}= \begin{cases}3 & \text { if } n=3 ; \\ 4 & \text { if } n \geqslant 4 .\end{cases}$

\section{Replacement product of Cayley graphs}

In this section, we investigate the restricted edge-connectivity of the replacement product of two Cayley graphs by a semidirect product of two groups. We will further confirm that under certain conditions on the underlying groups and generating sets, the replacement product of two Cayley graphs is indeed a Cayley graph. Using this result, we will give a necessary and sufficient condition for such Cayley graphs to be $\lambda^{\prime}$-optimal. Based on this condition, we will construct an example to answer Problem 1.4.

We first recall the notion of semidirect product of two groups. Let $A=(A, \circ)$ and $B=(B, *)$ be two finite groups. A group homomorphism from $A$ to $B$ is a mapping $\phi: A \rightarrow B$ satisfying $\phi(a \circ b)=\phi(a) * \phi(b)$. Let $e_{A}$ and $e_{B}$ be identities in $A$ and $B$, respectively, throughout this section. Group homomorphisms have two important and useful properties.

Proposition 5.1 Let $A$ and $B$ be two finite groups, and $\phi$ be a group homomorphism from $A$ to $B$. Then

(a) $\phi\left(e_{A}\right)=e_{B}$;

(b) $\phi\left(a^{-1}\right)=(\phi(a))^{-1}$ for any $a \in A$.

An action of $B$ on $A$ is a group homomorphism $\phi: B \rightarrow A u t(A)$ defined by $\phi(b)=\phi_{b}$ and $\phi\left(b_{1} b_{2}\right)=\phi\left(b_{1}\right) \phi\left(b_{2}\right)=\phi_{b_{1}} \phi_{b_{2}}$.

The orbit of $a \in A$ under the action $\phi$ of $B$ is expressed as $a^{B}=\left\{\phi_{b}(a) \in A: b \in B\right\}$.

Example 5.2 Let $A=\left(\mathbb{Z}_{2}\right)^{n}, B=\mathbb{Z}_{n}$, and let $e_{i}$ be an element in $A$ defined in (2.1) for each $i=0,1, \ldots, n$. 
The action $\phi$ of $B$ on $A$ is defined as follows. For each $a=a_{1} a_{2} \ldots a_{n} \in A$,

$$
\phi_{i}(a)=a_{1-i} a_{2-i} \ldots a_{n-i(\bmod n)} \text { for each } i=0, \ldots, n-1 \in B .
$$

For example, if $a=e_{1}$, then $\phi_{i}\left(e_{1}\right)=e_{i+1}$ for each $i=0,1, \ldots, n-1$. Under $\phi$ the orbit $e_{1}^{B}=\left\{e_{1}, e_{2}, \cdots, e_{n}\right\}$.

We now introduce the concept of the semidirect product of two finite groups following Robison [29].

The (external) semidirect product $A \rtimes_{\phi} B$ of groups $A$ and $B$ with respect to $\phi$ is the group with set $A \times B=\{(a, b): a \in A, b \in B\}$ and binary operation "*"

$$
\left(a_{1}, b_{1}\right) *\left(a_{2}, b_{2}\right)=\left(a_{1} \phi_{b_{1}}\left(a_{2}\right), b_{1} b_{2}\right) \text { for any } a_{1}, a_{2} \in A \text { and } b_{1}, b_{2} \in B \text {. }
$$

The identity is $\left(e_{A}, e_{B}\right)$. Since $\phi_{b} \in \operatorname{Aut}(A)$ is an automorphism from $A$ to $A$, by Proposition 5.1 (a)

$$
\phi_{b}(a)=e_{A} \Leftrightarrow a=e_{A} \text { for any } a \in A \text { and } b \in B,
$$

By (5.1), it is easy to verify that the inverse $(a, b)^{-1}$ of $(a, b)$ is $\left(\phi_{b^{-1}}\left(a^{-1}\right), b^{-1}\right)$, that is,

$$
(a, b)^{-1}=\left(\phi_{b^{-1}}\left(a^{-1}\right), b^{-1}\right) .
$$

It is also easy to check that the set $\left\{\left(a, e_{B}\right): a \in A\right\}$ forms a normal subgroup of $A \rtimes_{\phi} B$ isomorphic to $A$, and the set $\left\{\left(e_{A}, b\right): b \in B\right\}$ forms a subgroup of $A \rtimes_{\phi} B$ isomorphic to $B$. Thus, $A \rtimes_{\phi} B \cong A \rtimes B$, a semidirect product of two subgroups $A$ and $B$ of a group $\Gamma$, where $A$ is normal.

The direct product $A \times B$ is a special case of $A \rtimes_{\phi} B$, in which the action $\phi(b)$ is the identity automorphism of $A$ for any $b \in B$, and so $\left(a_{1}, b_{1}\right) *\left(a_{2}, b_{2}\right)=\left(a_{1} a_{2}, b_{1} b_{2}\right)$. Thus the semidirect product is a generalization of the direct product of two groups.

Many groups can be expressed as a semidirect product of two groups. For example, using the semidirect product, Feng [8] and Ganesan [9] determined the automorphism groups of some Cayley graphs generated by transposition sets; Zhou [38] determined the full automorphism group of the alternating group graph. The semidirect product of groups is also used to prove that some networks are Cayley graphs. For example, using the semidirect product, Zhou et al. [39] showed that the dual-cube $D C_{n}$ is a Cayley graph $C_{(\Gamma \times \Gamma) \rtimes_{\phi} \mathbb{Z}_{2}}(S)$, where $\Gamma=\left(\mathbb{Z}_{2}\right)^{n}$, the action $\phi: \mathbb{Z}_{2} \rightarrow \operatorname{Aut}(\Gamma \times \Gamma)$ is defined by

$$
\phi_{i}(\alpha, \beta)= \begin{cases}(\alpha, \beta) & \text { if } i=0 \\ (\beta, \alpha) & \text { if } i=1\end{cases}
$$

and $S=\left\{\left(e_{0}, e_{1}, 0\right), \ldots,\left(e_{0}, e_{n}, 0\right),\left(e_{0}, e_{0}, 1\right)\right\}$.

Assumption 5.3 Let $A$ and $B$ be two groups with generating sets $S_{A}$ and $S_{B}$, respectively, $\left|S_{A}\right|=|B| \geqslant 2, \phi$ be such an action of $B$ on $A$ that $S_{A}=x^{B}$ for some $x \in S_{A}$, and $S=\left\{\left(e_{A}, b\right): b \in S_{B}\right\} \cup\left\{\left(x, e_{B}\right)\right\}$.

Theorem 5.4 Under Assumption 5.3, S generates $A \rtimes_{\phi} B$. Moreover, if $S_{B}=S_{B}^{-1}$ and $x=x^{-1}$, then $S=S^{-1}$ and $C_{A \rtimes_{\phi} B}(S)$ is a replacement product of $C_{A}\left(S_{A}\right)$ and $C_{B}\left(S_{B}\right)$. 
Remark 5.5 Before proving this result, we make some remarks on the theorem.

(a) Since Cayley graphs under our discussion are undirected, by the definition of Cayley graphs, it is clear that the conditions " $S_{A}=S_{A}^{-1}, S_{B}=S_{B}^{-1}$ and $S=S^{-1}$ " are necessary to guarantee that Cayley graphs $C_{A}\left(S_{A}\right), C_{B}\left(S_{B}\right)$ and $C_{A \rtimes_{\phi} B}(S)$ are undirected. By Proposition 5.1 (b) for any action $\phi$ of $B$ on $A$,

$$
\left(x, e_{B}\right)^{-1}=\left(\phi_{e_{B}}\left(x^{-1}\right), e_{B}\right)=\left(x^{-1}, e_{B}\right) .
$$

Thus, the condition " $S=S^{-1}$ " means that

$$
\begin{aligned}
\left\{\left(e_{A}, b\right): b \in S_{B}\right\} \cup\left\{\left(x, e_{B}\right)\right\} & =\left(\left\{\left(e_{A}, b\right): b \in S_{B}\right\} \cup\left\{\left(x, e_{B}\right)\right\}\right)^{-1} \\
& =\left\{\left(e_{A}, b^{-1}\right): b \in S_{B}\right\} \cup\left\{\left(x^{-1}, e_{B}\right)\right\}
\end{aligned}
$$

which implies that the condition " $S=S^{-1}$ " is equivalent to the condition " $S_{B}=$ $S_{B}^{-1}$ and $x=x^{-1 "}$.

Furthermore, since $S_{A}=x^{B}$ under the action $\phi$, for any $a \in S_{A}$, there is some $b \in B$ such that $a=\phi_{b}(x)$. By Proposition 5.1 (b) we have that

$$
x=x^{-1} \Leftrightarrow a=\phi_{b}(x)=\phi_{b}\left(x^{-1}\right)=\left(\phi_{b}(x)\right)^{-1}=a^{-1} \text { for any } a \in S_{A} .
$$

(b) The original and simple statement of Theorem 5.4 is due to Alon et al. (see Theorem 2.3 in [1], as a special case of zig-zag products without proof), and a comparatively complete statement is given by Hoory et al. (see Theorem 11.22 in [13]) without the conditions " $x=x^{-1}$ and $S_{B}=S_{B}^{-1}$ ", and with an unperfect proof. We give a complete proof here.

Proof. By the explanation in Remark 5.5 (a), we only need to prove that $S$ generates $A \rtimes_{\phi} B$ and $C_{A \rtimes_{\phi} B}(S)$ is a replacement product of $C_{A}\left(S_{A}\right)$ and $C_{B}\left(S_{B}\right)$.

We first show that $S$ generates $A \rtimes_{\phi} B$. To the end, we only need to show that any $(a, b) \in A \rtimes_{\phi} B$ can be expressed as products of a sequence of elements of $S$.

By the hypothesis, $S_{A}$ is a generating set of $A$ and is the orbit $x^{B}$ of some $x \in S_{A}$ under the action $\phi$ of $B$ on $A$. Since $(a, b)=\left(a, e_{B}\right) *\left(e_{A}, b\right)$, it can be written as a product of elements from the set $\left\{\left(s_{a}, e_{B}\right): s_{a} \in S_{A}\right\} \cup\left\{\left(e_{A}, s_{b}\right): s_{b} \in S_{B}\right\}$. Since $S_{A}=x^{B}$, for $s_{a} \in S_{A}$ there is some $b \in B$ such that $s_{a}=\phi_{b}(x)$, where $b$ can be expressed as products of a sequence of elements of $S_{B}$ since $S_{B}$ is a generating set of $B$ by the hypothesis. Also since for any $b \in B$ and $\phi_{b}(x) \in S_{A}$,

$$
\left(s_{a}, e_{B}\right)=\left(\phi_{b}(x), e_{B}\right)=\left(e_{A}, b\right) *\left(x, e_{B}\right) *\left(e_{A}, b^{-1}\right),
$$

the element $\left(s_{a}, e_{B}\right)$ can be expressed as products of a sequence of elements of $S$. This implies that $S$ generates the group $A \rtimes_{\phi} B$.

We now show that $C_{A \rtimes_{\phi} B}(S)$ is a replacement product of $C_{A}\left(S_{A}\right)$ and $C_{B}\left(S_{B}\right)$. By Remark 5.5, under Assumption 5.3, Cayley graphs $C_{A}\left(S_{A}\right), C_{B}\left(S_{B}\right)$ and $C_{A \rtimes_{\phi} B}(S)$ are well-defined and undirected, and so satisfy the requirements in Definition 2.4. 
Let $(y, i)$ and $(z, j)$ be two distinct vertices in $C_{A \rtimes_{\phi} B}(S)$, where $y, z \in A=V\left(C_{A}\left(S_{A}\right)\right)$ and $i, j \in B=V\left(C_{B}\left(S_{B}\right)\right)$. Since $C_{A \rtimes_{\phi} B}(S)$ is a Cayley graph, we have that

$$
\begin{aligned}
(y, i)(z, j) \in E\left(C_{A \rtimes_{\phi} B}(S)\right) & \Leftrightarrow(y, i)^{-1} *(z, j) \\
& =\left(\phi_{i^{-1}}\left(y^{-1}\right), i^{-1}\right) *(z, j) \\
& =\left(\phi_{i^{-1}}\left(y^{-1}\right) \phi_{i^{-1}}(z), i^{-1} j\right) \\
& =\left(\phi_{i^{-1}}\left(y^{-1} z\right), i^{-1} j\right) \\
& \in S=\left\{\left(e_{A}, b\right): b \in S_{B}\right\} \cup\left\{\left(x, e_{B}\right)\right\} .
\end{aligned}
$$

If $\left(\phi_{i^{-1}}\left(y^{-1} z\right), i^{-1} j\right) \in\left\{\left(e_{A}, b\right): b \in S_{B}\right\}$, then $y=z$ by (5.1), and $i j \in E\left(C_{B}\left(S_{B}\right)\right)$, which means that the edge $(y, i)(y, j)$ of $C_{A \rtimes_{\phi} B}(S)$ is an edge in $C_{A}\left(S_{A}\right) \circledR C_{B}\left(S_{B}\right)$.

If $\left(\phi_{i^{-1}}\left(y^{-1} z\right), i^{-1} j\right)=\left(x, e_{B}\right)$, then $i=j$ and $\phi_{i^{-1}}\left(y^{-1} z\right)=x$. Since $\phi_{i^{-1}} \phi_{i}=$ $\phi\left(i^{-1}\right) \phi(i)=\phi\left(i^{-1} i\right)=\phi\left(e_{B}\right)$ is the identity automorphism of $A$, we have $\phi_{i^{-1}}^{-1}=\phi_{i}$. Thus, $y^{-1} z=\phi_{i^{-1}}^{-1}(x)=\phi_{i}(x) \in x^{B}=S_{A}$, that is, $z=y \phi_{i}(x)$ and $y z \in E\left(C_{A}\left(S_{A}\right)\right)$. Therefore, if we use $e_{y}^{i}$ and $e_{z}^{i}$ to label the edge $y z \in C_{A}\left(S_{A}\right)$ for each $(y, i)(z, i) \in E\left(C_{A \rtimes_{\phi} B}(S)\right)$, that is $y z=e_{y}^{i}=e_{z}^{i}$, then the edge $(y, i)(z, i)$ of $C_{A \rtimes_{\phi} B}(S)$ is an edge in $C_{A}\left(S_{A}\right) \AA C_{B}\left(S_{B}\right)$.

It follows that the structure of $C_{A \rtimes_{\phi} B}(S)$ satisfies the requirements of Definition 2.4, and so $C_{A \rtimes_{\phi} B}(S)$ is a replacement product of $C_{A}\left(S_{A}\right)$ and $C_{B}\left(S_{B}\right)$.

Example 5.6 Let $A=\left(\mathbb{Z}_{2}\right)^{n}$ and $B=\mathbb{Z}_{n}$. Then $e_{A}=e_{0}$ and $e_{B}=0$. Let $S_{A}=$ $\left\{e_{1}, e_{2}, \ldots, e_{n}\right\}$, where $e_{i}$ is defined in (2.1), and $e_{i}^{-1}=e_{i}$ for each $i \in\{1,2, \ldots, n\}$, and let $S_{B}= \pm\left\{s_{1}, s_{2}, \ldots, s_{k}\right\}$. The Cayley graph $C_{A}\left(S_{A}\right)$ is a hypercube $Q_{n}$ by Example 2.3 and the Cayley graph $C_{B}\left(S_{B}\right)$ is a circulant graph $G(n, \pm S)$ by Example 2.2. Let $\phi$ be the action of $B$ on $A$ defined in Example 5.2. Then $S_{A}$ is the orbit $e_{1}^{B}$ of $e_{1} \in S_{A}$ under $\phi$. Let $S=\left\{\left(e_{A}, s\right): s \in S_{B}\right\} \cup\left\{\left(e_{1}, e_{B}\right)\right\}$. Then $S=S^{-1}$. By Theorem 5.4, $S$ generates $A \rtimes_{\phi} B$, and $C_{A \rtimes_{\phi} B}(S)$ is a replacement product of $C_{A}\left(S_{A}\right)$ and $C_{B}\left(S_{B}\right)$.

In special, if $S_{B}=\{1, n-1\}$, then $S=\left\{\left(e_{0}, 1\right),\left(e_{0}, n-1\right),\left(e_{1}, 0\right)\right\}$. The Cayley graph $C_{\left(\mathbb{Z}_{2}\right)^{n} \rtimes_{\phi} \mathbb{Z}_{n}}(S)=Q_{n} \mathbb{R} C_{n}=C C C_{n}$. The cube-connected cycle $C C C(3)$, shown on the right side in Figure 4 , is a replacement product of $Q_{3}$ and $C_{3}$, and is the Cayley graph $C_{\mathbb{Z}_{2}^{3} \rtimes_{\phi} \mathbb{Z}_{3}}(\{(000,1),(000,2),(100,0)\})$.

A graph $G$ is $\kappa$-optimal if $\kappa(G)=\delta(G)$. The following theorem presents a necessary and sufficient condition for a Cayley graph $C_{A \rtimes_{\phi} B}(S)$ to be $\lambda^{\prime}$-optimal if $C_{A}\left(S_{A}\right)$ is $\kappa$ optimal and $C_{B}\left(S_{B}\right)$ is $\lambda^{\prime}$-optimal.

Theorem 5.7 Under Assumption 5.3, let $S=\left\{\left(e_{A}, s\right): s \in S_{B}\right\} \cup\left\{\left(x, e_{B}\right)\right\}$ and $S=S^{-1}$. If Cayley graph $C_{A}\left(S_{A}\right)$ is $\kappa$-optimal and Cayley graph $C_{B}\left(S_{B}\right)$ is $\lambda^{\prime}$-optimal, then Cayley graph $C_{A \rtimes_{\phi} B}(S)$ is $\lambda^{\prime}$-optimal $\Leftrightarrow\left|S_{A}\right| \geqslant 2\left|S_{B}\right|$.

Proof. By Theorem 5.4, $C_{A \rtimes_{\phi} B}(S)$ is a replacement product of $C_{A}\left(S_{A}\right)$ and $C_{B}\left(S_{B}\right)$. Since $C_{A}\left(S_{A}\right)$ is $\kappa$-optimal, $\kappa\left(C_{A}\left(S_{A}\right)\right)=\lambda\left(C_{A}\left(S_{A}\right)\right)=\delta\left(C_{A}\left(S_{A}\right)\right)=\left|S_{A}\right| \geqslant 2$. Also since $C_{B}\left(S_{B}\right)$ is $\lambda^{\prime}$-optimal, by Corollary 4.7 (a) $C_{A \rtimes_{\phi} B}(S)$ is $\lambda^{\prime}$-optimal if and only if $\left|S_{A}\right| \geqslant 2\left|S_{B}\right|$. 
Example 5.8 By Example 5.6, the cube-connected cycle $C C C_{n}=Q_{n} \AA C_{n}$ is 3-regular, $\xi\left(C C C_{n}\right)=4,\left|S_{Q_{n}}\right|=n \geqslant 2$ and $\left|S_{C_{n}}\right|=2$.

$$
\left|S_{A}\right|= \begin{cases}3<4=2\left|S_{B}\right| & \text { if } n=3 \\ n \geqslant 4=2\left|S_{B}\right| & \text { if } n \geqslant 4\end{cases}
$$

By Example 4.9 and Theorem [5.7, $C C C_{n}$ is

$$
\left\{\begin{array}{l}
\text { not } \lambda^{\prime} \text {-optimal }\left(\lambda^{\prime}=\lambda=3<4=\xi\right) \text { if } n=3 \\
\left.\lambda^{\prime} \text {-optimal (i.e., } \lambda^{\prime}=4=\xi\right) \text { if } n \geqslant 4
\end{array}\right.
$$

Theorem 5.9 Let $A=\left(\mathbb{Z}_{2}\right)^{n}$ and $B=\mathbb{Z}_{n}, S_{A}=\left\{e_{1}, e_{2}, \ldots, e_{n}\right\}$, where $e_{i}$ is defined in (2.1), $S_{B}= \pm\left\{s_{1}, s_{2}, \ldots, s_{k}\right\}$ with $k \geqslant 2$ and $s_{k}<\frac{n}{2}$, $\phi$ be the action of $B$ on $A$ defined in Example 5.2. Let $G=C_{A \rtimes_{\phi} B}(S)$ with order $v(G)$, where $S=\left\{\left(e_{0}, s\right): s \in S_{B}\right\} \cup\left\{\left(e_{1}, 0\right)\right\}$. If $\frac{n}{2}<\left|S_{B}\right|<n-1$, then $G$ is not $\lambda^{\prime}$-optimal, and

$$
\lambda(G)<\lambda^{\prime}(G)=n<\frac{v(G)}{2} \text { for } n \geqslant 3
$$

and $G[X] \cong C_{B}\left(S_{B}\right)$ for any $\lambda^{\prime}$-atom $X$ of $G$.

Proof. By Example $2.3 C_{A}\left(S_{A}\right) \cong Q_{n}$, by Example $2.2 C_{B}\left(S_{B}\right) \cong G\left(n ; S_{B}\right)$, and by Theorem 5.4 the Cayley graph $G=C_{A \rtimes_{\phi} B}(S)$ is a replacement product of $Q_{n}$ and $G\left(n ; S_{B}\right)$. Since $k \geqslant 2$ and $s_{k}<\frac{n}{2}, G\left(n ; S_{B}\right)$ is $\lambda^{\prime}$-optimal by Example 2.2. Since $Q_{n}$ is $\kappa$-optimal and $\left|S_{A}\right|=n<2\left|S_{B}\right|, G$ is not $\lambda^{\prime}$-optimal by Theorem 5.7. By Corollary 4.5, $\lambda^{\prime}(G)=\min \left\{n, 2\left|S_{B}\right|\right\}=n$. Since $G$ is vertex-transitive and $\left|S_{B}\right|<n-1$, we have that

$$
\lambda(G)=\delta(G)=|S|=\left|S_{B}\right|+1<n=\lambda^{\prime}(G) .
$$

Note that $v(G)=n \cdot 2^{n}$ and that $k \geqslant 2$ implies $n \geqslant 5$. It follows that

$$
\lambda(G)<\lambda^{\prime}(G)=n=\frac{n 2^{n}}{2^{n}}=\frac{v(G)}{2^{n}}<\frac{v(G)}{2} \text { for } n \geqslant 3 .
$$

We now show the second conclusion. Let $X$ be a $\lambda^{\prime}$-atom of $G$ and $F=E_{G}(X)$. Then $|X| \leqslant \frac{v(G)}{2}$ and $F$ is a $\lambda^{\prime}$-cut of $G$. We need to prove $G[X] \cong C_{B}\left(S_{B}\right)$. We first note that

$$
|F|=\lambda^{\prime}(G)=n<2\left|S_{B}\right|=4 k .
$$

Let $\left\{X_{1}, X_{2}, \ldots, X_{n}\right\}$ be a partition of $V(G)$ satisfied property in Proposition [2.5. Then $G\left[X_{i}\right] \cong C_{B}\left(S_{B}\right)$ for each $i \in I_{2^{n}}$. If there exists some $j \in I_{2^{n}}$ such that $G\left[X_{j}\right]$ is disconnected in $G-F$ then, by Lemma 4.3 and Example 2.2 ,

$$
\begin{aligned}
|F| & \geqslant \min \left\{\kappa\left(C_{A}\left(S_{A}\right)\right)+\lambda\left(C_{B}\left(S_{B}\right)\right)-1,2 \lambda\left(C_{B}\left(S_{B}\right)\right), \lambda^{\prime}\left(C_{B}\left(S_{B}\right)\right)+2\right\} \\
& =\min \{n+2 k-1,4 k\}=4 k,
\end{aligned}
$$

which contradicts with (5.3) . It follows that $G\left[X_{i}\right]$ is connected in $G-F$, that is, either $X_{i} \subset X$ or $X_{i} \subset \bar{X}$ for each $i \in I_{2^{n}}$. 
If both $X$ and $\bar{X}$ contain at least two sets of $X_{1}, X_{2}, \ldots, X_{2^{n}}$, then, by comparing the structure of $G$ with that of $Q_{n}$, it is easy to see that the subset of edges in $Q_{n}$ corresponding to $F$ is a restricted edge-cut of $Q_{n}$. Hence, by Example 2.3,

$$
|F| \geqslant \lambda^{\prime}\left(Q_{n}\right)=2 n-2>n=\lambda^{\prime}(G)=|F|,
$$

a contradiction. Namely, $X=X_{i}$ or $\bar{X}=X_{i}$ for some $i \in I_{2^{n}}$.

Since $|X| \leqslant \frac{v(G)}{2}$, we have $X=X_{i}$ and $\bar{X}=V(G) \backslash X_{i}$ for some $i \in I_{2^{n}}$. Thus every $\lambda^{\prime}$-cut of $G$ isolates a subgraph which is isomorphic to $C_{B}\left(S_{B}\right)$. In other words, $G[X] \cong G\left[X_{i}\right] \cong C_{B}\left(S_{B}\right)$ for each $i \in I_{2^{n}}$.

Remark 5.10 We make some remarks on the conditions in Theorem 5.9.

The condition " $k \geqslant 2$ " is necessary. In fact, if $k=1$, then $C_{B}\left(S_{B}\right)$ is a cycle $C_{n}$. By Example 5.8,

$$
C C C_{n} \text { is }\left\{\begin{array}{l}
\text { not } \lambda^{\prime} \text {-optimal and } \lambda^{\prime}=\lambda=3 \text { if } n=3 ; \\
\lambda^{\prime} \text {-optimal if } n \geqslant 4 .
\end{array}\right.
$$

The condition " $\left|S_{B}\right|>\frac{n}{2}$ " is necessary. Theorem 5.7 means that $C_{A \rtimes_{\phi} B}(S)$ is

$$
\text { not } \lambda^{\prime} \text {-optimal } \Leftrightarrow\left|S_{A}\right|<2\left|S_{B}\right| \text {, i.e., }\left|S_{B}\right|>\frac{1}{2}\left|S_{A}\right|=\frac{1}{2} n \text {. }
$$

The condition " $\left|S_{B}\right|<n-1$ " is also necessary. In fact, if $\left|S_{B}\right|=n-1$ then $G\left(n ; S_{B}\right)$ is a complete graph $K_{n}$ by Example 2.2. Thus, $\lambda(G)=n=\lambda^{\prime}(G)$, which contradicts to our conclusion.

The following theorem gives a straight answer to Problem 1.4.

Theorem 5.11 For a given odd integer $d(\geqslant 5)$ and any integer $s$ with $1 \leqslant s \leqslant d-3$, there is a Cayley graph $G$ with degreed such that $\lambda^{\prime}(G)=d+s<\frac{1}{2} v(G)$.

Proof. In Theorem 5.9, let $n=d+s$ and $k=\frac{d-1}{2}$, then $\left|S_{B}\right|=d-1$ and $G=$ $C_{\mathbb{Z}_{2}^{d+s} \rtimes_{\phi} \mathbb{Z}_{d+s}}(S)$ is a Cayley graph. Since $1 \leqslant s \leqslant d-3$, we have $\frac{d+s}{2}<\left|S_{B}\right|<d+s-1$. By Theorem 5.9, $G$ is not $\lambda^{\prime}$-optimal, and

$$
\lambda(G)=d<\lambda^{\prime}(G)=d+s<\frac{(d+s) \cdot 2^{d+s}}{2}=\frac{v(G)}{2} .
$$

The theorem follows.

\section{Conclusion}

In this paper, we investigate the restricted edge-connectivity of replacement product of two graphs. By means of the semidirect product two groups, we further confirm that under certain conditions, the replacement product of two Cayley graphs is also a Cayley graph, and give a necessary and sufficient condition for such Cayley graphs to have maximum restricted edge-connectivity. Based on these results, for given odd integer $d$ and integer $s$ 
with $d \geqslant 5$ and $1 \leqslant s \leqslant d-3$, we construct a Cayley graph with degree $d$ whose restricted edge-connectivity is equal to $d+s$, which answers a problem proposed ten years ago.

In the proof of this result, the replacement product of graphs plays a key role. Thus, further properties of replacement products deserve further research.

Acknowledgements This work was supported by National Natural Science Foundation of China (Grant Nos. 61272008, 11571044), University Natural Science Research Project of Anhui Province (Grant No. KJ2016A003) and Scientific Research Fund of Anhui University of Finance \& Economics (Grant No. ACKY1532).

\section{References}

[1] N. Alon, A. Lubotzky, and A. Wigderson, Semi-direct product in groups and zig-zag product in graphs: connections and applications (extended abstract). In 42nd IEEE Symposium on Foundations of Computer Science (Las Vegas, NV, 2001), pp. 630-637. IEEE Computer Society, 2001.

[2] A. Donno, Replacement and zig-zag products, Cayley graphs and Lamplighter random walk. Int. J. Group Theory, 2(1) (2013), 11-35.

[3] J. E. Dunbar and T.W. Haynes, Domination in inflated graphs, Congr. Numer. 118 (1996), 143-154.

[4] A.H. Esfahanian, Generalized measures of fault tolerance with application to $n$-cube networks. IEEE Trans. Comput. 38 (11) (1989), 1586-1591.

[5] A.H. Esfahanian and S.L. Hakimi, On computing a conditional edge-connectivity of a graph. Inform. Process. Lett. 27 (1988), 195-199.

[6] O. Favaron, Irredundance in inflated graphs. Journal of Graph Theory, 28(2)(1998), 97-104.

[7] O. Favaron, Inflated graphs with equal independence number and upper irredundance number. Discrete Mathematics, 236(1-3) (2001), 81-94.

[8] Y.-Q. Feng, Automorphism groups of Cayley graphs on symmetric groups with generating transposition sets. Journal of Combinatorial Theory, Series B, 96 (2006), 67-72.

[9] A. Ganesan, Automorphism groups of Cayley graphs generated by connected transposition sets, Discrete Mathematics, 313 (2013), 2482-2485.

[10] M. Gromov, Filling Riemannian manifolds. J. Differential Geom. 81 (1983), 1-147.

[11] A. Hellwig and L. Volkmann, Sufficient conditions for $\lambda_{r}$-optimality in graphs of diameter 2. Discrete Mathematics, 283 (1-3) (2004), 113-120.

[12] A. Hellwig and L. Volkmann, Sufficient conditions for graphs to be $\lambda_{r}$-optimal, superedge-connected, and maximally edge-connected. Journal of Graph Theory, 48 (3) (2005), $228-246$.

[13] S. Hoory, N. Linial and A. Wigderson, Expander graphs and their applications. Bull. Amer. Math. Soc. (N.S.) 43(4) (2006), 439-561. 
[14] L.-Y. Kang, M. Y. Sohn and T.C.E. Cheng, Paired-domination in inflated graphs. Theoretical Computer Science, 320 (2004), 485-494.

[15] C. Kelley, J. Rosenthal and D. Sridhara, Some new algebraic constructions of codes from graphs which are good expanders. in "Proc. of the 41-st Allerton Conference on Communication, Control and Computing," (2003), 1280-1289.

[16] C. Kelley, D. Sridhara and J. Rosenthal, Zig-Zag and Replacement product graphs and LDPC codes. Advances in Mathematics of Communications, 2(4)(2008), 347-372.

[17] F. Li, W. Wang, Z. Xu and H. Zhao, Some results on the lexicographic product of vertextransitive graphs. Applied Mathematics Letters, 24 (2011), 1924-1926.

[18] L. Li and J.-M. Xu, On restricted edge-connectivity of vertex-transitive graphs. Journal of University of Science and Technology of China, 34 (3) (2004), 266-272.

[19] Q.L. Li and Q. Li, Reliability analysis of circulant graphs. Networks, 31(2) (1998), 61-65.

[20] Q.L. Li and Q. Li, Super edge connectivity properties of connected edge symmetric graphs. Networks, 33 (2) (1999), 147-159.

[21] J. Liu and X.-D. Zhang, Cube-connected complete graphs. IAENG International Journal of Applied Mathematics, 44(3)(2014), IJAM_44_3_03.

[22] Q.M. Malluhi and M.A. Bayoumi, The hierarchical hypercube: A new interconnection topology for massively parallel systems. IEEE Trans. Parallel Distrib. Syst. 5(1) (1994), $17-30$.

[23] J. Meng, Optimally super-edge-connected transitive graphs. Discrete Mathematics, 260 (13) (2003), 239-248.

[24] J. Meng and Y. Ji, On a kind of restricted edge connectivity of graphs. Discrete Applied Mathematics, 117 (1-3) (2002), 183-193.

[25] J.-P. Ou and F.-J. Zhang, Super restricted edge connectivity of regular graphs. Graphs and Combinatorics, 21 (4) (2005), 459-467.

[26] F.P. Preparata and J. Vuillemin, The cube-connected cycles: a versatile network for parallel computation. Communications of the Association for Computing Machinery, 24 (5) (1981), 300-309.

[27] J. Puech, The lower irredundance and domination parameters are equal for in ated trees. J. Combin. Math. Combin. Comput. 33 (2000), 117-127.

[28] O. Reingold, S. Vadhan, and A. Wigderson, Entropy waves, the zig-zag graph product, and new constant-degree expanders. Ann. of Math. (2), 155(1) (2002), 157-187.

[29] D.J.S. Robinson, An Introduction to Abstract Algebra. Walter de Gruyter, 2003, pp. 75-76.

[30] N. Ueffing and L. Volkmann, Restricted edge-connectivity and minimum edge-degree. Ars Combinatoria, 66 (2003), 193-203.

[31] M. Wang and Q. Li, Conditional edge connectivity properties, reliability comparison and transitivity of graphs. Discrete Mathematics, 258 (1-3) (2002), 205-214. 
[32] Y.-Q. Wang and Q. Li, Super-edge-connectivity properties of graphs with diameter 2. Journal of Shanghai Jiaotong University (Science), 33 (6) (1999), 646-649.

[33] Y.-Q. Wang and Q. Li, An Ore type sufficient condition for a graph to be super restricted edge-connected. Journal of Shanghai Jiaotong University, 35 (8) (2001), 1253-1255.

[34] J.-M. Xu, Topological Structure and Analysis of Interconnection Networks. Kluwer Academic Publishers, Dordrecht/Boston/London, 2001.

[35] J.-M. Xu, Theory and Application of Graphs. Kluwer Academic Publishers, Dordrecht/Boston/London, 2003.

[36] J.-M. Xu, Review and prospect of study on combinatorial networks (in Chinese). http://staff.ustc.edu.cn/ xujm/essay2003b.pdf, 2003.

[37] J.-M. Xu and K.-L. Xu, On restricted edge-connectivity of graphs. Discrete Mathematics, 243(1-3) (2002), 291-298.

[38] J.-X. Zhou, The automorphism group of the alternating group graph. Applied Mathematics Letters, 24 (2011), 229-231.

[39] S.-M. Zhou, L.-X. Chen and J.-M. Xu, Conditional fault diagnosability of dual-cubes. International Journal of Foundations of Computer Science. 23 (8) (2012), 1729-1749. 